\title{
Linear Multihop Amplify-and-Forward Relay Channels: Error Exponent and Optimal Number of Hops
}

Hien Quoc Ngo and Erik G. Larsson

\section{Linköping University Post Print}

N.B.: When citing this work, cite the original article.

(C2011 IEEE. Personal use of this material is permitted. However, permission to reprint/republish this material for advertising or promotional purposes or for creating new collective works for resale or redistribution to servers or lists, or to reuse any copyrighted component of this work in other works must be obtained from the IEEE.

Hien Quoc Ngo and Erik G. Larsson, Linear Multihop Amplify-and-Forward Relay Channels: Error Exponent and Optimal Number of Hops, 2011, IEEE Transactions on Wireless Communications, (10), 11, 3834-3842. http://dx.doi.org/10.1109/TWC.2011.092011.102194 


\title{
Linear Multihop Amplify-and-Forward Relay Channels: Error Exponent and Optimal Number of Hops
}

\author{
Hien Quoc Ngo and Erik G. Larsson
}

\begin{abstract}
We compute the random coding error exponent for linear multihop amplify-and-forward (AF) relay channels. Instead of considering only the achievable rate or the error probability as a performance measure separately, the error exponent results can give us insight into the fundamental tradeoff between the information rate and communication reliability in these channels. This measure enables us to determine what codeword length that is required to achieve a given level of communication reliability at a rate below the channel capacity. We first derive a general formula for the random coding exponent of general multihop AF relay channels. Then we present a closedform expression of a tight upper bound on the random coding error exponent for the case of Rayleigh fading. From the exponent expression, the capacity of these channels is also deduced. The effect of the number of hops on the performance of linear multihop AF relay channels from the error exponent point of view is studied. As an application of the random coding error exponent analysis, we then find the optimal number of hops which maximizes the communication reliability (i.e., the random coding error exponent) for a given data rate. Numerical results verify our analysis, and show the tightness of the proposed bound.
\end{abstract}

Index Terms-Amplify-and-forward multihop relaying, linear multihop relay channel, random coding error exponent.

\section{INTRODUCTION}

In a multihop relay channel, a source node communicates with a destination node with the help of a number of relay nodes. This channel is believed to improve the system performance because of the reduction in the transmission distance per hop which directly translates into improved channel gains and hence improved communication reliability. Therefore, there has been significant previous research in this field [1]-[3]. In [1], the outage probability of multihop relay channels with non-regenerative relays over Nakagami$m$ fading channels was analyzed. Bounds on the end-to-end signal-to-noise ratio (SNR) of multihop transmissions with non-regenerative relays over Nakagami- $m$ fading channels and with non-regenerative blind relays over generalized fading channels were proposed in [2] and [3], respectively. Then, the outage probability and the average bit-error probability

Manuscript received December 10, 2010; revised June 20, 2011; accepted August 23, 2011. The associate editor coordinating the review of this paper and approving it for publication was Giuseppe Abreu. This work was supported in part by the Swedish Research Council (VR) and ELLIIT. E. Larsson is a Royal Swedish Academy of Sciences (KVA) Research Fellow supported by a grant from the Knut and Alice Wallenberg Foundation.

H. Q. Ngo and E. G. Larsson are with the Department of Electrical Engineering (ISY), Linköping University, 58183 Linköping, Sweden (Email: nqhien@isy.liu.se; egl@isy.liu.se)

Digital Object Identifier xxx/xxx were studied. However, there have been few works that consider the fundamental tradeoff between the communication reliability and transmission rate for arbitrary SNRs. Instead of considering the error probability or the achievable rate as separate performance measures, the random coding error exponent (RCEE) results can provide insight into the fundamental tradeoff between these measures. The RCEE for discrete channels was first introduced by Gallager [4]. It has been then derived for point-to-point transmission over fading channels for single-antenna systems in [5], [6], and MIMO systems in [7]. Recently, the RCEE has been considered for relay channels, including the dual-hop relay channel [8] and the two-way relay channel [9].

Intuitively, the larger number of hops that the system uses, the better is the performance in terms of link reliability. However, when the number of hops increases, more time slots (or frequencies) have to be spent on the transmission, leading to a reduction of spectral efficiency. Therefore, there has been an increasing interest in investigating the effects of the number of hops on the performance of multihop relay channels. In [2], the authors showed that the performance in terms of average bit error probability and outage probability degraded with an increase in the number of hops. However, the authors assumed that the average SNRs per hop were equal when the number of hops changed, and the signal attenuation due to path loss between the nodes was not considered. The performance of one-hop and two-hop decode-and-forward relay channels was compared in [10]. The channel model in [10] assumed attenuation due to the path loss and Rayleigh fading. By considering the outage probability, the authors showed that one-hop transmission outperforms two-hop transmission at high spectral efficiency. When the number of hops is greater than 2, the linear (or one-dimensional) multihop relay channel is of great interest because it is tractable to analyze and is considered as a special case of a more general two-dimensional network. In [11], a linear multihop relay network for AWGN channels was considered. The authors determined the optimum number of hops that achieves the desired end-to-end rate with the smallest possible transmission power. In [12], an infrastructure-based fixed multihop relay network with a regenerative protocol was considered under the assumptions that the channel coefficients were constant and that all links had the same average path loss exponent. The authors showed that at high SNR, single-hop transmission has better spectral efficiency than $n$-hop transmission, and vice versa at low SNR. In addition, the optimal number of 
hops was considered from a spectral efficiency perspective. The rate of the linear mutihop relay channel was analyzed to show merits of multihop relaying schemes in broadband cellular mesh networks [13]. The authors in [13] showed that at low SNR, multihop with a large number of hops offers improved performance under the path-loss effect. In [14], a linear multihop relay network over a slow-fading channel was considered. An upper bound on the outage probability was derived. Then, the optimal number of hops that minimizes the end-to-end outage probability was investigated. However, most of these works considered outage probability, error probability, or achievable rate as performance measure when studying the effect of the number of hops. Since the RCEE captures the tradeoff between the communication reliability and the data rate, the RCEE is a more fundamental and objective performance measure for comparing and investigating the effect of the number of hops in multihop relay channels.

In this paper, we consider a linear multihop amplify-andforward (AF) relay channel where all relay terminals are located on a straight line from the source to the destination for simplicity. ${ }^{1}$ To investigate the tradeoff between the communication reliability and transmission rate, we first develop an expression for the RCEE of general multihop AF relay channels. Then, we derive a closed-form expression of a tight upper bound on the RCEE assuming Rayleigh fading. From this expression, the capacity is also deduced. Finally, we consider the optimal number of hops which maximizes the RCEE for a given data rate.

The remainder of this paper is organized as follows. In Section II, we provide the mathematical preliminaries for the development of the RCEE for general multihop AF relay channels. The optimal number of hops in terms of the RCEE for linear multihop relay channels is developed in Section III. In Section IV, some numerical results are provided. Finally, concluding remarks are given in Section V.

Notation: We shall use the following notation. For a random variable $X$, we use $p_{X}(x)$ and $\mathbb{E}_{X}(\cdot)$ to denote the probability density function (PDF) of $X$ and the expected value of $X$, respectively. A circularly symmetric complex Gaussian distribution with mean $\mu$ and variance $\sigma^{2}$ is denoted by $\mathcal{C N}\left(\mu, \sigma^{2}\right)$. We use $\mathrm{A} \rightarrow \mathrm{B}$ to represent the communication link from $\mathrm{A}$ to $\mathrm{B}$.

\section{Mathematical Preliminaries}

Consider a multihop AF relay channel with $K$ hops as in Fig. 1a, consisting of a source terminal $\mathrm{T}_{1}$, a destination terminal $\mathrm{T}_{K+1}$ and $K-1$ relay terminals $\mathrm{T}_{k}, k=2, . ., K$. The transmitted powers at the source and the relay terminals $T_{k}$ are denoted by $p_{k}, k=1, . ., K$. We assume that a terminal node cannot transmit and receive signals simultaneously. This setup is referred to as "half duplex". Furthermore, to guarantee that there is no interference between the different hops, the transmission is performed via time-division where the transmission from the source to the destination is divided into $K$ time slots. In the $k$ th time slot, the signal received

\footnotetext{
${ }^{1}$ Throughout, we will use the term "linear multihop relay channel" to denote "linear multihop AF relay channel".
}

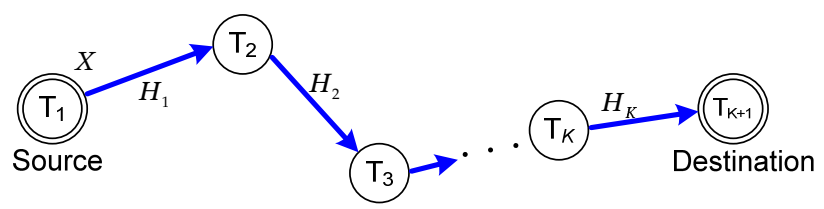

(a)

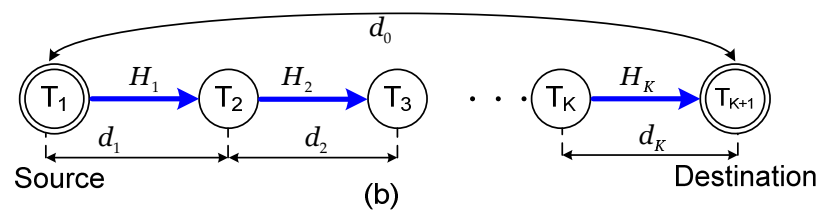

Fig. 1. (a) Multihop amplify-and-forward relay channel; (b) Linear multihop relay channel.

by the $k$ th intermediate terminal is amplified with a relaying gain $G_{k}$ and then forwarded to the $(k+1)$ th terminal. It is also assumed that the $k$ th terminal can only hear the signal transmitted by the $(k-1)$ th terminal in the $(k-1)$ th time slot. This is logical in a scenario where the $k$ th terminal may be busy or engaged in data transmission to another terminal during the previous time slots.

Denote by $H_{k}$ the channel coefficient from $\mathrm{T}_{k}$ to $\mathrm{T}_{k+1}$. Then $H_{k}$ 's are statistically mutually independent provided that the terminals are sufficiently well spatially separated. The received signal at the destination is given by

$$
\begin{aligned}
Y=\left(\prod_{k=2}^{K} G_{k}\right) & \left(\prod_{k=1}^{K} H_{k}\right) X+\left(\prod_{k=2}^{K} G_{k}\right)\left(\prod_{k=2}^{K} H_{k}\right) Z_{1} \\
& +\left(\prod_{k=3}^{K} G_{k}\right)\left(\prod_{k=3}^{K} H_{k}\right) Z_{2}+\ldots+Z_{K}
\end{aligned}
$$

where $X$ is the signal transmitted from the source, and $Z_{k} \sim$ $\mathcal{C N}\left(0, N_{0}\right)$ is the complex additive Gaussian noise at $\mathrm{T}_{k+1}$.

The reliability function or error exponent corresponding to rate $R$ for a channel of capacity $C \geq R$ is the best decay exponent in the codeword length $N$ of the average error probability that one can achieve [4]. That is,

$$
E(R)=\lim _{N \rightarrow \infty} \sup -\frac{1}{N} \ln P_{e}^{\text {opt }}(R, N)
$$

where $P_{e}^{\mathrm{opt}}(R, N)$ denotes the average block error probability for the best block code of length $N$ and rate $R$ for a given channel. $^{2}$

From (1), using Gallager's random coding results [4, Theorem 5.6.2], we can bound the average probability of a decoding error over the ensemble of $(N, R)$ block codes for multihop relay channels according to (3), shown at the bottom of next page, where $\rho \in[0,1]$ is an arbitrary number and where the transition PDF $p_{Y \mid X, H_{1}, \ldots, H_{K}}\left(y \mid x, h_{1}, \ldots, h_{K}\right)$ is given by

$$
\begin{aligned}
& p_{Y \mid X, H_{1}, \ldots, H_{K}}\left(y \mid x, h_{1}, \ldots, h_{K}\right) \\
& =\frac{1}{\pi \sigma^{2}} \exp \left\{-\frac{\left|y-\left(G_{2} \ldots G_{K}\right)\left(h_{1} \ldots h_{K}\right) x\right|^{2}}{\sigma^{2}}\right\}
\end{aligned}
$$

\footnotetext{
${ }^{2}$ Throughout the paper, rate will be measured in units of nats per second per $\mathrm{Hz}($ nats $/ \mathrm{s} / \mathrm{Hz})$.
} 
and where

$$
\sigma^{2} \triangleq\left(\sum_{i=2}^{K} \prod_{k=i}^{K} G_{k}^{2}\left|H_{k}\right|^{2}+1\right) N_{0}
$$

and $M \geq 2$ is the number of codewords of length $N$.

Since the source and destination communicate each codeword in $K N$ symbol-time intervals using $K$-hop transmission, the transmission rate $R$ in nats $/ \mathrm{s} / \mathrm{Hz}$ is

$$
R=\frac{\ln M}{K N}
$$

leading to

$$
M=\left\lceil e^{K N R}\right\rceil
$$

where $\lceil z\rceil$ denotes the smallest integer greater than or equal to $z$. Therefore, from (3) and (7), we obtain

$$
P_{e} \leq \exp \left\{-N\left[E_{0}\left(\rho, p_{X}\right)-\rho K R\right]\right\}
$$

where $E_{0}\left(\rho, p_{X}\right)$ is given by (9), shown at the bottom of the page.

Since the input distribution from which the ensemble of codebooks is constructed is arbitrary in (8), we can obtain a tighter bound on the average probability of a decoding error as follows:

$$
E_{\mathrm{r}}(R)=\max _{0 \leq \rho \leq 1} \max _{p_{X}(x)}\left\{E_{0}\left(\rho, p_{X}(x)\right)-\rho K R\right\} .
$$

$E_{\mathrm{r}}(R)$ in (10) is the RCEE. It is generally very difficult (if not impossible) to cope with the double maximization in (10) since the inner integral is raised to a fractional exponent when $\rho \in(0,1)$ and owing to the lack of knowledge about the optimal input distribution $p_{X}(x)$. Therefore, a Gaussian input distribution $p_{X}(x)$ is often assumed for analytical tractability [5]-[9], i.e., $p_{X}(x)$ is given by

$$
p_{X}(x)=\frac{1}{\pi p_{1}} \exp \left(-\frac{|x|^{2}}{p_{1}}\right) .
$$

The choice of a Gaussian input gives a lower bound on the random coding exponent but it still would provide a better error exponent behavior than a practical input distribution. This is the reason for why in many papers which considered the RCEE, the Gaussian input distribution is often assumed. By using the Gaussian input distribution, we obtain the following proposition for the RCEE of multihop AF relay channels.

Proposition 1: With the Gaussian input distribution $p_{X}(x)$, the RCEE of the multihop AF relay channel is given by

$$
E_{\mathrm{r}}(R)=\max _{0 \leq \rho \leq 1}\left\{E_{0}(\rho)-\rho K R\right\}
$$

where

$$
E_{0}(\rho)=-\ln \mathbb{E}_{\gamma_{\text {end }}}\left\{\left(1+\frac{\gamma_{\text {end }}}{1+\rho}\right)^{-\rho}\right\}
$$

and $\gamma_{\text {end }}$ is the end-to-end SNR of multihop relay channels given by

$$
\gamma_{\text {end }}=\frac{\left|H_{1}\right|^{2} p_{1} \prod_{i=2}^{K} G_{i}^{2}\left|H_{i}\right|^{2}}{\left(\sum_{i=2}^{K} \prod_{k=i}^{K} G_{k}^{2}\left|H_{k}\right|^{2}+1\right) N_{0}} .
$$

Proof: See Appendix A.

Remark 1: The factor $K$ of $\rho R$ in (12) is due to the use of $K$ time slots for the transmission in multihop AF relay channels. In the special case of $K=2$, (12) becomes the formula for RCEE of dual-hop relay channels that we derived in [8].

Remark 2: From results in [4], we deduce the following properties of the RCEE $E_{\mathrm{r}}(R)$. Let $\rho_{\text {opt }}$ be the optimal value of $\rho$ which maximizes $E_{0}(\rho)-\rho K R$ in (12). Then

$$
\left.\left[\frac{\partial E_{0}(\rho)}{\partial \rho}\right]\right|_{\rho=\rho_{\mathrm{opt}}}-K R=0 .
$$

Since $\partial E_{0}(\rho) / \partial \rho$ is decreasing with respect to $\rho$, the optimal value $\rho_{\text {opt }}$ lies in $[0,1]$ if

$R_{\mathrm{cr}}=\left.\frac{1}{K}\left[\frac{\partial E_{0}(\rho)}{\partial \rho}\right]\right|_{\rho=1} \leq R \leq\left.\frac{1}{K}\left[\frac{\partial E_{0}(\rho)}{\partial \rho}\right]\right|_{\rho=0}=\langle C\rangle$

where $R_{\mathrm{cr}}$ and $\langle C\rangle$ are the critical rate and the (ergodic) capacity for the AF multihop relay channel, respectively. For $R<R_{\mathrm{cr}}, E_{0}(\rho)-\rho K R$ is maximized (over $\rho \in[0,1]$ ) by $\rho_{\text {opt }}=1$, yielding $E_{\mathrm{r}}(R)=E_{0}(1)-K R$. The slope of the exponent-rate curve is equal to $-K$. For $R>\langle C\rangle$, $E_{0}(\rho)-\rho K R$ is maximized when $\rho_{\text {opt }}=0$, yielding $E_{\mathrm{r}}(R)=0$.

\section{ERRor EXPONENT ANALYSIS AND OPTIMAL NUmber of Hops For Linear Multihop Relay Channels}

In this section, we investigate the effect of the number of hops on the performance of linear multihop relay channels from the RCEE point of view. The model that we use for the propagation channel $H_{k}, k=1, \ldots, K$, includes fast fading (Rayleigh fading) and geometric (path loss) attenuation. We first propose an upper bound on the end-to-end SNR. Closed-form expressions for the RCEE and the capacity are then deduced. Finally, we maximize the error exponent by optimizing the number of hops.

$$
P_{e} \leq(M-1)^{\rho}\left\{\int_{h_{K}} p_{H_{K}}\left(h_{K}\right) \cdots \int_{h_{1}} p_{H_{1}}\left(h_{1}\right) \int_{y}\left[\int_{x} p_{X}(x) p_{Y \mid X, H_{1}, \ldots, H_{K}}\left(y \mid x, h_{1}, \ldots, h_{K}\right)^{\frac{1}{1+\rho}} d x\right]^{1+\rho} d y d h_{1} \cdots d h_{K}\right\}^{N}
$$

$$
E_{0}\left(\rho, p_{X}\right)=-\ln \left\{\int_{h_{K}} p_{H_{K}}\left(h_{K}\right) \cdots \int_{h_{1}} p_{H_{1}}\left(h_{1}\right) \int_{y}\left[\int_{x} p_{X}(x) p_{Y \mid X, H_{1}, \ldots, H_{K}}\left(y \mid x, h_{1}, \ldots, h_{K}\right)^{\frac{1}{1+\rho}} d x\right]^{1+\rho} d y d h_{1} \ldots d h_{K}\right\}
$$




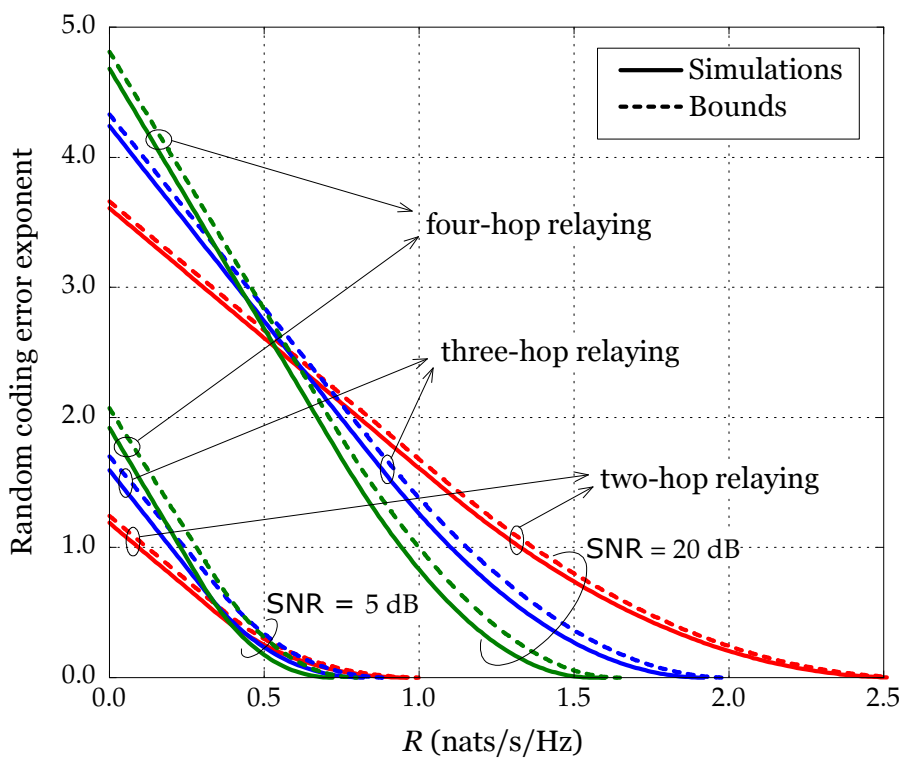

Fig. 2. Random coding error exponent of linear multihop relay channels and its bound from Theorem 1 . Here $K=2,3,4, \beta=4$ and SNR $=5,20 \mathrm{~dB}$.

\section{A. System Model}

We consider a linear (one-dimensional) multihop relay channel as in Fig. 1b where all relay terminals $T_{k}, k=2, \ldots, K$, are located uniformly spaced along the straight line from the source terminal $T_{1}$ to the destination terminal $T_{K+1}$. This kind of channel is a somewhat simplified model of the real world, but we can find it in practical systems, for example in vehicular communications (e.g., the communication between cars on a highway), or the communication between roadside units located along the road [15], [16]. Furthermore, it can be a building block of more general two-dimensional networks. Therefore, there has been much research on such linear networks [11]-[14], [17].

There are two kinds of AF relaying. The first one is fixedgain relaying in which the relaying gain is fixed for all fading states. This protocol reduces the implementation complexity in terms of channel state information (CSI) [2], [18]. The second one is CSI-assisted relaying in which the relaying gain is calculated based on the preceding channel state. There is much interest in this protocol since it is not complex to implement and it has higher performance compared with the fixed-gain relaying protocol. For CSI-assisted relaying, the relaying gain at $\mathrm{T}_{k}$ is $G_{k}^{2}=\frac{p_{k}}{p_{k-1}\left|H_{k-1}\right|^{2}+N_{0}}$, where $p_{k}$ is the transmitted power at $\mathrm{T}_{k}$, which yields an end-toend SNR of an intractable form as in [1]. Another choice of relaying gain is $G_{k}^{2}=\frac{p_{k}}{p_{k-1}\left|H_{k-1}\right|^{2}}$, which represents and ideal/hypothetical AF relaying, and renders an end-to-end SNR that has an analytically more tractable form [2], [19]-[21]. This ideal/hypothetical AF relaying requires a slightly different power constraint at each relay terminal. However, it provides a benchmark for all practical CSI-assisted AF relaying schemes since it gives a very tight upper bound on the exact form of CSI-assisted AF relaying. For this reason, here we consider multihop channels with ideal/hypothetical AF relaying. For ideal/hypothetical AF relaying, the end-to-end equivalent SNR

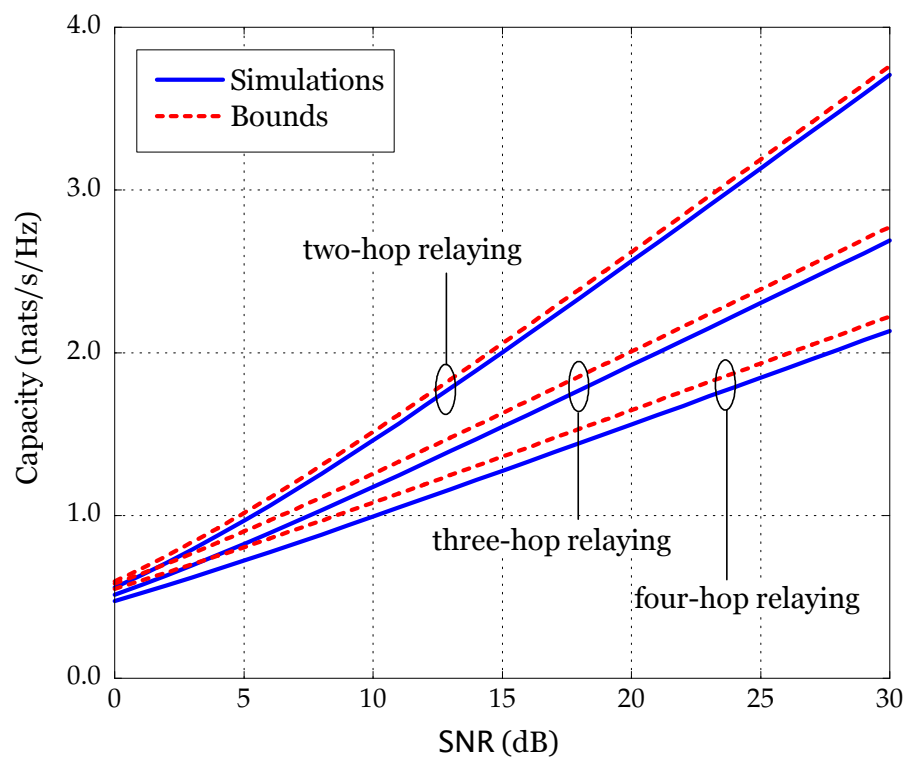

Fig. 3. Capacity of linear multihop relay channels, for $K=2,3,4$, and $\beta=4$.

of multihop channels is given by [19]

$$
\gamma_{\mathrm{end}}=\left(\sum_{k=1}^{K} \frac{1}{\gamma_{k}}\right)^{-1}
$$

where $\gamma_{k} \triangleq \frac{p_{k}\left|H_{k}\right|^{2}}{N_{0}}$ is the instantaneous SNR of the link $T_{k} \rightarrow T_{k+1}$.

We use a total power constraint, i.e., $\sum_{k=1}^{K} p_{k}=P$. To perform optimal power allocation, each terminal must know the CSI of all hops, which leads to a high operational complexity. Therefore, we consider equal power allocation here, i.e., $p_{k}=P / K$ for $k=1,2, \ldots, K$.

Denote with $d_{k}$ the distance between $T_{k}$ and $T_{k+1}, k=$ $1,2, \ldots, K$, and let $\epsilon_{k} \triangleq d_{k} / d_{0}$ be the ratio of $d_{k}$ to $d_{0}$, where $d_{0}$ is the distance between the source and the destination. Since the relay terminals are located with equal spacing on the line between the source and the destination, $\epsilon_{k}=\frac{1}{K}$. We assume that there is geometric path loss and that the channel is Rayleigh fading with $\Omega_{k}$ being the average squared magnitude of $H_{k}$. Then, $\gamma_{k}$ is exponentially distributed, with the PDF

$$
p_{\gamma_{k}}(\gamma)=\frac{1}{\bar{\gamma}_{k}} \exp \left(-\frac{\gamma}{\bar{\gamma}_{k}}\right)
$$

where $\bar{\gamma}_{k}$ is the average SNR of the $T_{k} \rightarrow T_{k+1}$ link, which is given by

$$
\bar{\gamma}_{k}=\frac{p_{k} \Omega_{k}}{N_{0}}=\frac{p_{k} \Omega_{0}}{N_{0} \epsilon_{k}^{\beta}}=\frac{P \Omega_{0}}{N_{0}} K^{\beta-1}
$$

where $\Omega_{0}$ is the mean of the squared amplitude of the channel coefficient for the link from the source to the destination, $\left|H_{0}\right|^{2} . \beta$ is the path loss exponent, which depends on the propagation environment and can range normally from 2 (propagation in free space) to about 4 (or even higher) in urban environments. 


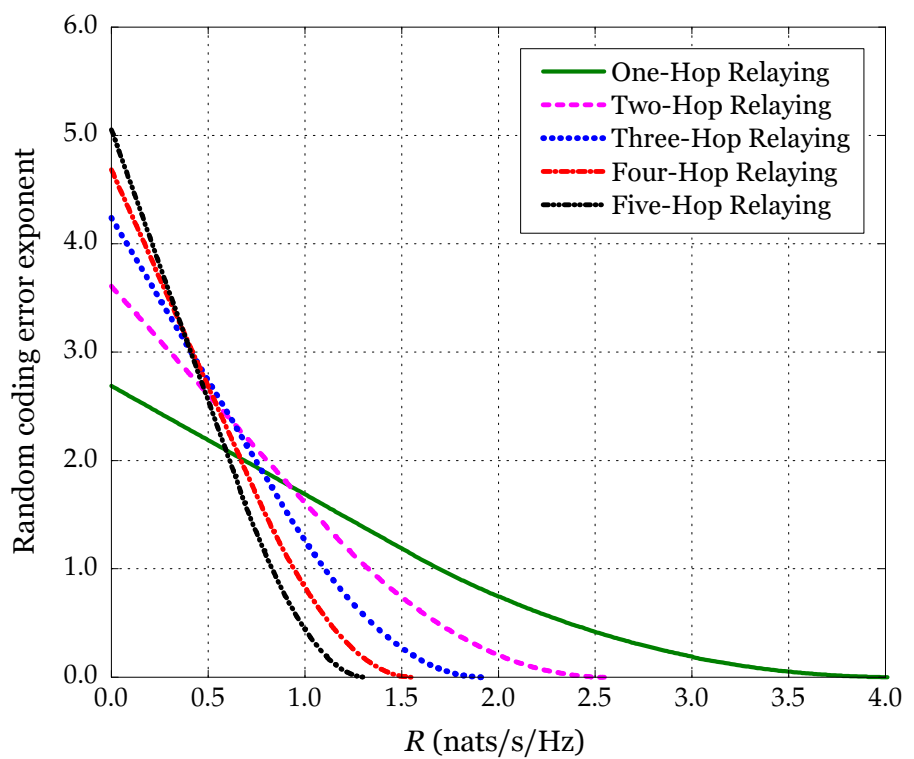

Fig. 4. Random coding error exponent of linear multihop relay channels, for $K=1,2,3,4$, and $5, \beta=4$ and $\mathrm{SNR}=20 \mathrm{~dB}$.

\section{B. Closed-form Solution for the RCEE of Linear Multihop Relay Channels}

From Proposition 1, it is difficult to derive the exact RCEE of linear multihop relay channels due to the analytically intractable form of the end-to-end SNR in (17). To allow an analytical derivation, we consider an upper bound on this endto-end SNR. ${ }^{3}$ Observing that the end-to-end SNR is dominated by the most noisy hop, we could upper bound $\gamma_{\text {end }}$ by the minimum of all $\gamma_{k}, k=1,2, \ldots, K$. However, this bound is not tight in case the values of $\gamma_{1}, \gamma_{2}, \ldots$, and $\gamma_{K}$ are close to each other. For this case, using the Cauchy-Schwarz inequality will give a tighter upper bound. As a result, we provide an upper bound on the end-to-end SNR which combines the two bounds just discussed and which is tight for all cases, as follows:

$$
\gamma_{\mathrm{end}}=\left(\sum_{k=1}^{K} \frac{1}{\gamma_{k}}\right)^{-1} \leq \gamma_{\mathrm{b}} \triangleq \min \left(\gamma_{\mathrm{b}, 1}, \gamma_{\mathrm{b}, 2}\right)
$$

where $\gamma_{\mathrm{b}, 1}=\min \left(\gamma_{1}, \gamma_{2}, \ldots, \gamma_{K}\right)$ represents the upper bound obtained by using the most noisy hop, and $\gamma_{\mathrm{b}, 2}=\frac{\gamma_{1}+\gamma_{2}+\ldots \gamma_{K}}{K^{2}}$ is the upper bound obtained by using the Cauchy-Schwarz inequality. To further analyze the RCEE, we first derive the cumulative density function (CDF) and PDF of $\gamma_{b}$ in the following proposition.

Proposition 2: The cumulative density function (CDF) and PDF of $\gamma_{b}$ in case of Rayleigh fading channels are given

\footnotetext{
${ }^{3}$ By using the well-known inequality between the geometric and arithmetic means, an upper bound on the end-to-end SNR of multihop channels with ideal/hypothetical AF relaying was proposed in [2] and [3]. However, if the values of $\gamma_{1}, \gamma_{2}, \ldots, \gamma_{K}$ are vastly different, this bound will not be tight.
}

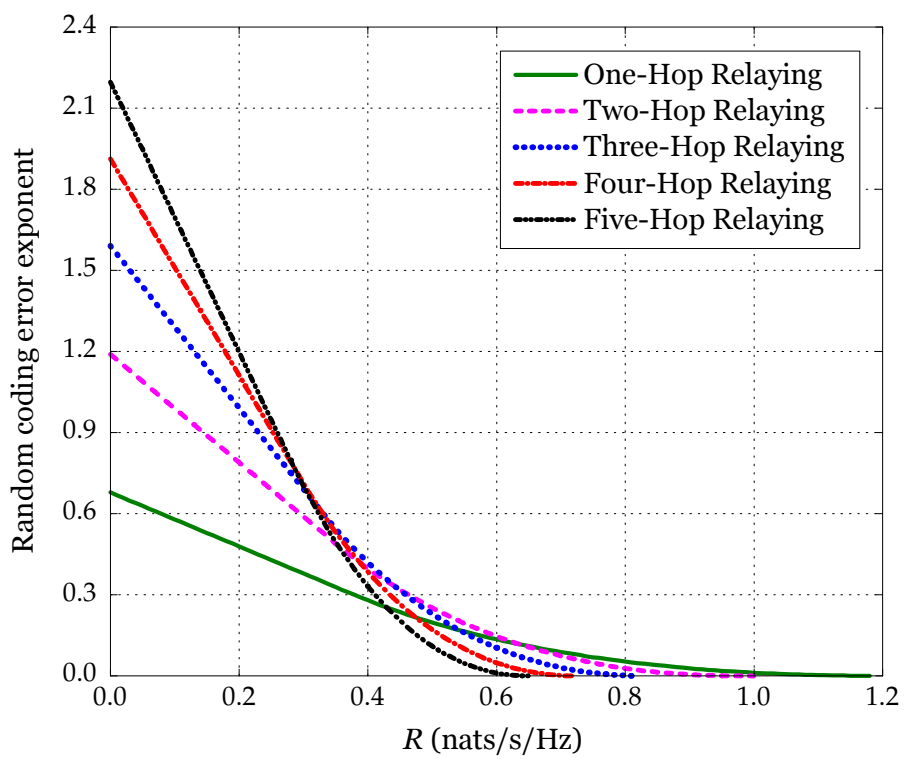

Fig. 5. Random coding error exponent of linear multihop relay channels, for $K=1,2,3,4$, and $5, \beta=4$ and $\mathrm{SNR}=5 \mathrm{~dB}$.

respectively by

$$
\begin{aligned}
F_{\gamma_{\mathrm{b}}}(\gamma)= & 1-\sum_{k=0}^{K-1} \frac{\left(K^{2}-K\right)^{k}}{k ! \bar{\gamma}^{k}} \gamma^{k} e^{-K^{2} \gamma / \bar{\gamma}} \\
p_{\gamma_{\mathrm{b}}}(\gamma)= & \sum_{k=0}^{K-2} \frac{\left(K^{2}-K\right)^{k} K}{k ! \bar{\gamma}^{k+1}} \gamma^{k} e^{-K^{2} \gamma / \bar{\gamma}} \\
& +\frac{\left(K^{2}-K\right)^{K-1} K^{2}}{(K-1) ! \bar{\gamma}^{K}} \gamma^{K-1} e^{-K^{2} \gamma / \bar{\gamma}}
\end{aligned}
$$

where $\bar{\gamma} \triangleq \frac{P \Omega_{0}}{N_{0}} K^{\beta-1}$.

Proof: See Appendix B

Owing to the monotonicity of the ln function, we can obtain an upper bound on the RCEE in the following theorem.

Theorem 1: With the Gaussian input distribution $p_{X}(x)$, the upper bound on the RCEE of a linear multihop relay channel is given by

$$
\tilde{E}_{\mathrm{r}}(R)=\max _{0 \leq \rho \leq 1}\left\{\tilde{E}_{0}(\rho)-\rho K R\right\}
$$

where $\tilde{E}_{0}(\rho)=0$ for $\rho=0$ and

$$
\begin{aligned}
\tilde{E}_{0}(\rho) & =-\ln \mathbb{E}_{\gamma_{\mathrm{b}}}\left\{\left(1+\frac{1}{1+\rho} \gamma_{\mathrm{b}}\right)^{-\rho}\right\} \\
& =-\ln \left\{\sum_{k=0}^{K-2} \frac{(K-1)^{k}}{k ! K^{k+1} \Gamma(\rho)} G_{2,1}^{1,2}\left(\frac{K^{-2} \bar{\gamma}}{1+\rho} \mid \begin{array}{l}
-k, 1-\rho \\
0
\end{array}\right)\right. \\
+ & \left.\frac{(K-1)^{K-1}}{(K-1) ! K^{K-1} \Gamma(\rho)} G_{2,1}^{1,2}\left(\frac{K^{-2} \bar{\gamma}}{1+\rho} \mid \begin{array}{l}
1-K, 1-\rho \\
0
\end{array}\right)\right\},
\end{aligned}
$$

for $0<\rho \leq 1$

where $\Gamma(\cdot)$ is Euler's gamma function, and $G_{p, q}^{m, n}(\cdot)$ is Meijer's G-function [22, eq. (8.2.1.1)].

Proof: See Appendix C

Furthermore, based on the random coding exponent we can find the capacity. 


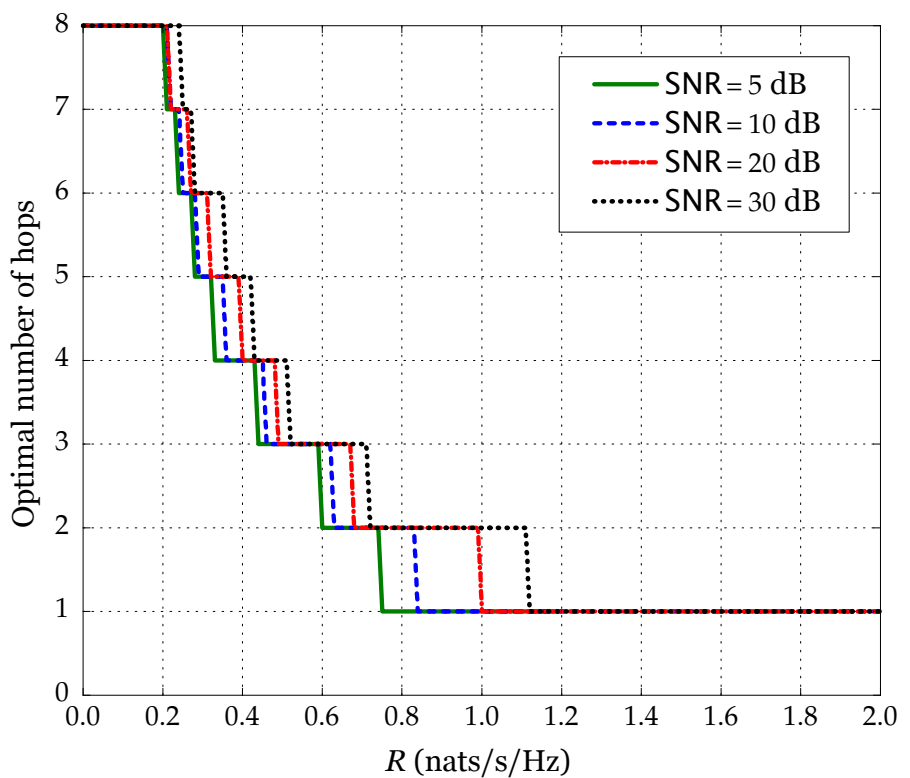

Fig. 6. Optimal number of hops, for $K_{\max }=8, \beta=4$ and $\mathrm{SNR}=5,10$, $20,30 \mathrm{~dB}$.

Corollary 1: The upper bound on the ergodic capacity of linear multihop relay channels is given by

$$
\begin{aligned}
\langle C\rangle= & \sum_{k=0}^{K-2} \frac{(K-1)^{k}}{k ! K^{k+1}} G_{3,2}^{1,3}\left(\frac{\bar{\gamma}}{K^{2}} \mid \begin{array}{l}
-k, 1,1 \\
1,0
\end{array}\right) \\
& +\frac{(K-1)^{K-1}}{(K-1) ! K^{K-1}} G_{3,2}^{1,3}\left(\begin{array}{l|l}
\frac{\bar{\gamma}}{K^{2}} & \begin{array}{l}
1-K, 1,1 \\
1,0
\end{array}
\end{array}\right) .
\end{aligned}
$$

Proof: See Appendix D.

\section{Optimal Number of Hops}

It is well-known that when the number of hops increases, the average SNR at each hop will increase due to the effect of path loss, but the spectral efficiency will decrease due to the need for using more time-slots (or frequencies). This reduction of the spectral efficiency is reflected by the factor $K$ in the term $\rho K R$ in (12). Therefore, depending on the data rate, the optimal number of hops which maximizes the RCEE will change correspondingly. The RCEE enables us to estimate the codeword length which is required to obtain a given error probability [6], [7]. Therefore, maximizing the error exponent is equivalent to maximizing the communication reliability with fixed coding complexity, or minimizing the required codeword length for a prescribed communication reliability.

In the following, we consider the optimal number of hops when the data rate is fixed. For a given data rate $R=R^{\star}$, the optimal number of hops which maximizes the RCEE (communication reliability) is determined by ${ }^{4}$

$$
\mathcal{P}= \begin{cases}\max _{K} & \tilde{E}_{\mathrm{r}}(R, K) \\ \text { s.t. } & R=R^{\star} \\ & 1 \leq K \leq K_{\max }\end{cases}
$$

\footnotetext{
${ }^{4}$ We add the parameter $K$ to the RCEE formula in (23) since we would like to find the optimal value of $K$.
}

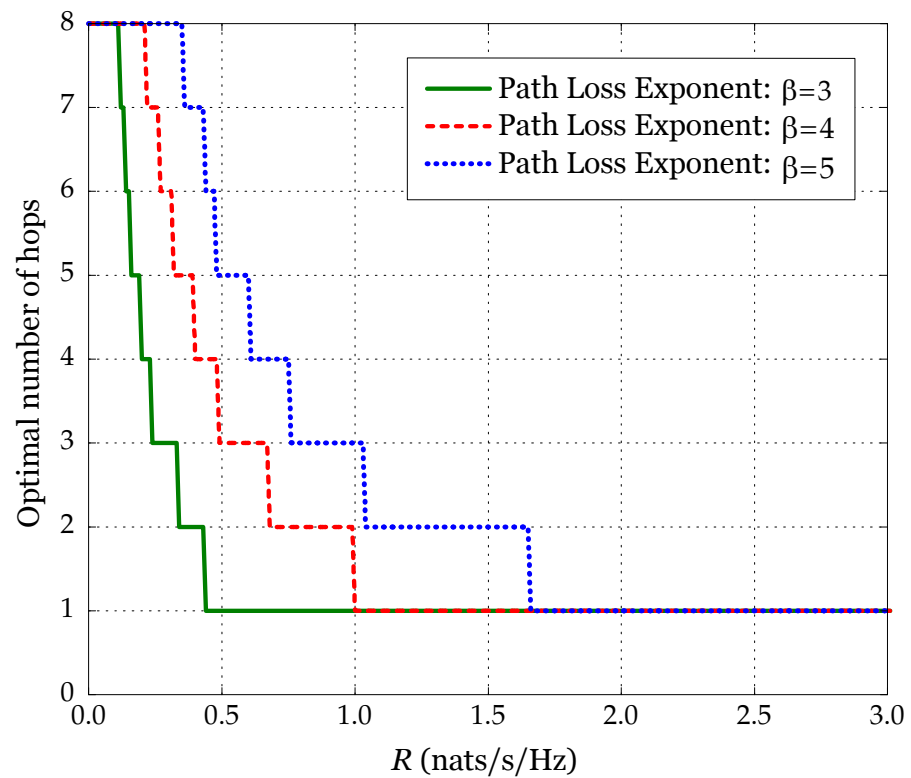

Fig. 7. Optimal number of hops, for $K_{\max }=8$, SNR $=20 \mathrm{~dB}$, and $\beta=3,4,5$.

where $K_{\max }$ is the maximal number of hops that the system can support.

The optimal number of hops can be easily found numerically using (26). For a given data rate $R=R^{\star}$, we first find the RCEE value $\tilde{E}_{\mathrm{r}}\left(R^{\star}, K\right)$ for different $K\left(1 \leq K \leq K_{\max }\right)$ using (23) and the method given in [23, Section 2.2.4]. We then find the optimal value of $K$ that maximizes $\tilde{E}_{\mathrm{r}}\left(R^{\star}, K\right)$.

\section{Numerical Results}

We present numerical results to verify our analysis. Throughout this section, we define SNR $\triangleq P \Omega_{0} / N_{0}$.

\section{A. Random Coding Error Exponent, Capacity and Their Bounds}

We first consider the tightness of our proposed bound. Fig. 2 shows simulation results of the RCEE versus $R$ for linear multihop relay channels and analytical results of the proposed bound in Theorem 1 at SNR $=5 \mathrm{~dB}$, and SNR $=20 \mathrm{~dB}$. We can see that our proposed bound provides a very tight bound on the error exponent. Furthermore, the lower the value of $K$ is, the tighter the proposed bounds are. This is due to the fact that our proposed bound will be tight if the probability is high that all $\gamma_{1}, \gamma_{2}, \ldots, \gamma_{K}$ are vastly different $\left(\gamma_{\mathrm{b}, 1}\right.$ provides a tight bound for this case) or close to each other $\left(\gamma_{\mathrm{b}, 2}\right.$ provides a tight bound for this case). This probability will decrease when $K$ increases.

The tightness of our proposed bound is also demonstrated in Fig. 3, which shows the simulation and analytical results for the ergodic capacity of linear multihop relay channels. Here, the bound is obtained by using Corollary 1 .

\section{B. The Effect of Number of Hops on the Random Coding Error Exponent}

We now consider the effect of the number of hops on the RCEE. Figs. 4 and 5 show the RCEE with different 
numbers of hops $K=1,2,3,4$, and 5 at SNR $=20$ and $5 \mathrm{~dB}$, respectively. It can be seen from these figures that for low rates, multihop relaying with large $K$ offers improved performance. ${ }^{5}$ By contrast, at high rates, multihop relaying with small $K$ is better. For example, at SNR $=20 \mathrm{~dB}$, and rate $R=1$ (nats $/ \mathrm{s} / \mathrm{Hz}$ ) (see Fig. 4), the error exponents are equal to $1.69,1.61,1.26,0.83$, and 0.44 for $K=1,2$, 3,4 , and 5 , respectively. These results imply that two-hop relaying, three-hop relaying, four-hop relaying, and five-hop relaying channels have to use respectively more than 1.04 , $1.34,2.03,3.84$ times the codeword length required for onehop relaying channels to achieve the same communication reliability at $R=1$ (see [6] for relation between the coding complexity and the RCEE). We can also see that for each rate value, there is an optimal number of hops $K$ that achieves the best performance in terms of RCEE. Furthermore, multihop relaying with a small number of hops is preferable for high SNR, while transmission with a large number of hops is better for low SNR. Similar conclusions were drawn in [11], [13].

\section{Optimal Number of Hops}

We give examples of the optimal number of hops based on the error exponent analysis. The optimal number of hops versus the rate $R$ is determined by using (26), and plotted in Figs. 6 and 7 for $K_{\max }=8$. Fig. 6 shows the optimal number of hops versus the data rate at $\beta=4$ for SNR $=5,10,20$ and $30 \mathrm{~dB}$. For example, for the values of $R$ equal to $0.2,0.4$, $0.6,0.8,1$, the optimal number of hops are respectively equal to $8,4,3,2,1$ at $\mathrm{SNR}=20 \mathrm{~dB}$, and $7,3,2,1,1$ at $\mathrm{SNR}=5$ $\mathrm{dB}$. We can see again that multihop relaying with a small $K$ is preferable in the high-rate regime and vice versa. Fig. 7 depicts the optimal number of hops versus the data rate at $\mathrm{SNR}=20 \mathrm{~dB}$, for $\beta=3,4$, and 5 . We can see that the larger the value of the path loss exponent is, the larger the number of hops is preferable. The reason is that when the path loss exponent is large, the SNR will dramatically decrease with the transmission distance, so we should use more hops to reduce the distance per hop, and vice versa for a low value of path loss exponent.

To ascertain the effectiveness of using the optimal number of hops in maximizing the error exponent, we compare the RCEE attained by using the optimal number of hops and the RCEE of a two-hop relaying channel. As can be seen from Fig. 8, using the optimal number of hops can significantly improve the RCEE. For example, at a data rate of $R=0.2$ (nats/s/Hz), the error exponents are respectively equal to 0.79 , 1.49 , and 3.21 for SNR $=5,10$, and $20 \mathrm{~dB}$ when using twohop relaying, compared to $1.26,2.19$, and 4.21 when using the optimal number of hops. These values reveal that to achieve the same communication reliability at $R=0.2$, the required codeword lengths when using the optimal number of hops can be reduced to about $63 \%, 68 \%$, and $76 \%$ of the corresponding lengths when using two-hop relaying for $\mathrm{SNR}=5,10$, and $20 \mathrm{~dB}$, respectively.

\footnotetext{
${ }^{5}$ Note that throughout the paper, "rate" refers to the effective rate taking into account the losses associated with orthogonality between the hops.
}

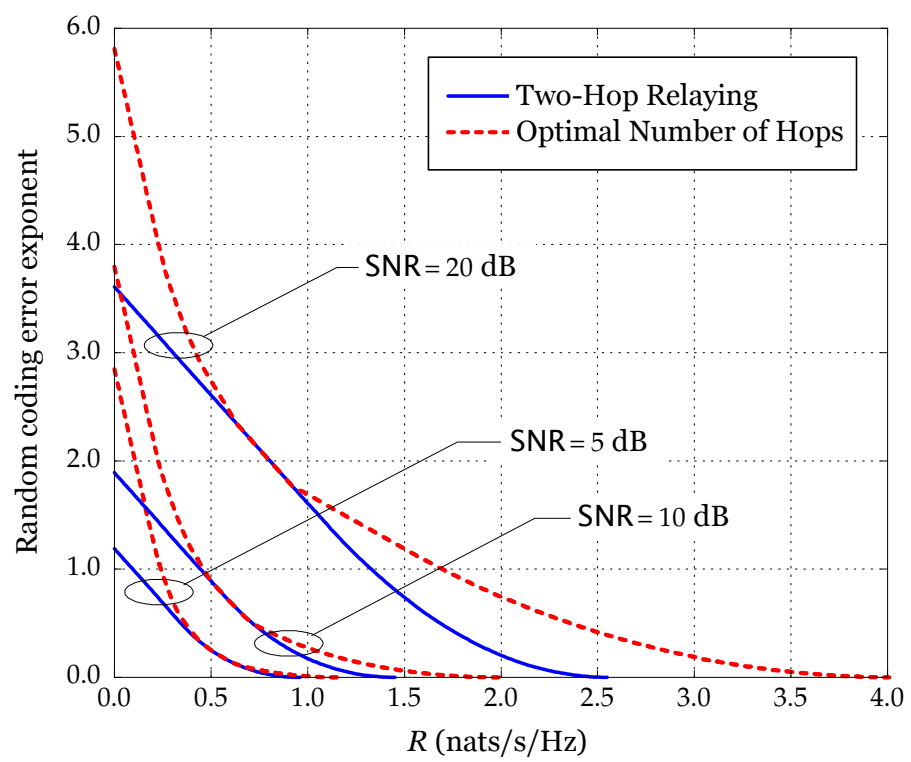

Fig. 8. Random coding error exponent for two-hop relaying and for the optimal number of hops, for $K_{\max }=8, \beta=4$ and SNR $=5,10,20 \mathrm{~dB}$.

\section{CONCLUSION}

We have considered the RCEE which characterizes the fundamental tradeoff between communication reliability and information rate in linear multihop AF relay channels. Based on the error exponent formula of general multihop AF relay channels, we presented a closed-form expression of a tight upper bound on the RCEE in terms of Meijer's G-function. From the error exponent expression, we also deduced the capacity and studied the effect of the number of hops on the performance.

We have found that multihop relaying with a small number of hops is preferable in the high-SNR regime, while transmission with a large number of hops is better for low SNR. Finally, we presented results on the optimal number of hops which maximizes the RCEE for a given data rate.

\section{APPENDIX}

\section{A. Proof of Proposition 1}

By substituting (4) and (11) into (9), we obtain (27), shown at the bottom of next page. Conditioned on $H_{1}, \ldots, H_{K}$, we first evaluate the integral over $x$ to obtain

$$
\begin{aligned}
& E_{0}\left(\rho, p_{X}\right)=-\ln \left\{\int_{h_{K}} p_{H_{K}}\left(h_{K}\right) \cdots \int_{h_{1}} p_{H_{1}}\left(h_{1}\right)\right. \\
& \times\left(\frac{1}{\pi \sigma^{2}}\right)^{\frac{1}{1+\rho}} \int_{y}\left[\frac{1+\rho}{1+\rho+\frac{p_{1}\left|\left(G_{2} \ldots G_{K}\right)\left(h_{1} \ldots h_{K}\right)\right|^{2}}{\sigma^{2}}}\right. \\
& \left.\left.\times \exp \left\{\frac{-|y|^{2} / \sigma^{2}}{1+\rho+\frac{p_{1}\left|\left(G_{2} \ldots G_{K}\right)\left(h_{1} \ldots h_{K}\right)\right|^{2}}{\sigma^{2}}}\right\}\right]^{1+\rho} d y d h_{1} \ldots d h_{K}\right\}
\end{aligned}
$$


and then over $y$ to obtain

$$
\begin{gathered}
E_{0}\left(\rho, p_{X}\right)=-\ln \left\{\int_{h_{K}} p_{H_{K}}\left(h_{K}\right) \cdots \int_{h_{1}} p_{H_{1}}\left(h_{1}\right)\right. \\
\left.\quad \times\left[\frac{1+\rho}{1+\rho+\frac{p_{1}\left|\left(G_{2} \ldots G_{K}\right)\left(h_{1} \ldots h_{K}\right)\right|^{2}}{\sigma^{2}}}\right]^{\rho} d h_{1} \ldots d h_{K}\right\} .
\end{gathered}
$$

Substituting (5) into (29), and defining $\gamma_{\text {end }}$ as in (14) completes the proof.

\section{B. Proof of Proposition 2}

From (20), the CDF of $\gamma_{b}$ is given by

$$
\begin{aligned}
& F_{\gamma_{\mathrm{b}}}(\gamma)=\operatorname{Pr}\left\{\min \left(\gamma_{1}, \gamma_{2}, \ldots, \gamma_{K}, \frac{\sum_{k=1}^{K} \gamma_{k}}{K^{2}}\right) \leq \gamma\right\} \\
& =1-\operatorname{Pr}\left\{\min \left(\gamma_{1}, \gamma_{2}, \ldots, \gamma_{K}, \frac{\sum_{k=1}^{K} \gamma_{k}}{K^{2}}\right) \geq \gamma\right\} \\
& =1-\operatorname{Pr}\left\{\gamma_{1} \geq \gamma, \gamma_{2} \geq \gamma, \ldots, \gamma_{K} \geq \gamma, \frac{\sum_{k=1}^{K} \gamma_{k}}{K^{2}} \geq \gamma\right\} .
\end{aligned}
$$

Let

$$
f_{K}(n, \gamma) \triangleq \operatorname{Pr}\left\{\gamma_{1} \geq \gamma, \gamma_{2} \geq \gamma, \ldots, \gamma_{K} \geq \gamma, \sum_{k=1}^{K} \gamma_{k} \geq n \gamma\right\}
$$

Then $f_{K}(n, \gamma)$ can be represented as (31), shown at the bottom of the page. Let

$$
\begin{array}{r}
g_{K}(n, \gamma) \triangleq \operatorname{Pr}\left\{\gamma_{1} \geq \gamma, \gamma_{2} \geq \gamma, \ldots, \gamma_{K} \geq n \gamma-\sum_{k=1}^{K-1} \gamma_{k},\right. \\
\left.\sum_{k=1}^{K-1} \gamma_{k} \leq(n-1) \gamma\right\} .
\end{array}
$$

Then (31) can be rewritten as

$$
f_{K}(n, \gamma)=e^{-\gamma / \bar{\gamma}_{K}} f_{K-1}(n-1, \gamma)+g_{K}(n, \gamma)
$$

We can see that (33) is a recursive formula, so we can obtain

$$
\begin{aligned}
& f_{K}(n, \gamma)=\exp \left(-\sum_{k=2}^{K} \frac{1}{\bar{\gamma}_{k}} \gamma\right) f_{1}(n-K+1, \gamma) \\
& +\sum_{k=2}^{K} \exp \left(-\sum_{i=k+1}^{K} \frac{1}{\bar{\gamma}_{i}} \gamma\right) g_{k}(n-K+k, \gamma)
\end{aligned}
$$

For $n \geq K, f_{1}(n-K+1, \gamma)=\exp \left(-\frac{n-K+1}{\bar{\gamma}_{k}} \gamma\right)$. Therefore, to find $f_{K}(n, \gamma)$, we first find $g_{K}(m, \gamma)$. We have

$$
\begin{aligned}
& g_{K}(m, \gamma)= \\
& \operatorname{Pr}\left\{\gamma \leq \gamma_{1} \leq(m-K+1) \gamma, \gamma \leq \gamma_{2} \leq(m-K+2) \gamma-\gamma_{1}, \ldots,\right. \\
& \left.\gamma \leq \gamma_{K-1} \leq(m-1) \gamma-\sum_{k=1}^{K-2} \gamma_{k}, m \gamma-\sum_{k=1}^{K-1} \gamma_{k} \leq \gamma_{K} \leq \infty\right\} \\
& =\int_{\gamma}^{(m-K+1) \gamma} \int_{\gamma}^{(m-K+2) \gamma-\gamma_{1}} \ldots \int_{\gamma}^{(m-1) \gamma-\sum_{k=1}^{K-2} \gamma_{k}} \\
& \quad \times \int_{m \gamma-\sum_{k=1}^{K-1} \gamma_{k}}^{\infty} \prod_{i=1}^{K} \frac{1}{\bar{\gamma}_{i}} e^{\gamma_{i} / \bar{\gamma}_{i}} d \gamma_{K} d \gamma_{K-1} \ldots d \gamma_{2} d \gamma_{1} .
\end{aligned}
$$

Replacing $\bar{\gamma}_{i}, i=1, \ldots, K$, by $\bar{\gamma}$, and then performing the integrations with respect to $\gamma_{K}, \gamma_{K-1}, \ldots, \gamma_{1}$, respectively, we obtain

$$
g_{K}(m, \gamma)=\frac{(m-K)^{K-1}}{(K-1) ! \bar{\gamma}^{K-1}} e^{-\frac{m}{\gamma} \gamma} \gamma^{K-1}
$$

Substituting (36) into (34), and using (30), we arrive at the desired result for the CDF of $\gamma_{b}$ as in Proposition 2. The PDF of $\gamma_{b}$ in (22) follows immediately by differentiating the CDF with respect to $\gamma$.

$$
\begin{aligned}
& E_{0}\left(\rho, p_{X}\right)=-\ln \left\{\int_{h_{K}} p_{H_{K}}\left(h_{K}\right) \cdots \int_{h_{1}} p_{H_{1}}\left(h_{1}\right) \frac{1}{\pi p_{1}}\left(\frac{1}{\pi \sigma^{2}}\right)^{\frac{1}{1+\rho}}\right. \\
& \left.\quad \times \int_{y}\left[\int_{x} \exp \left(-\frac{|x|^{2}}{p_{1}}\right) \exp \left\{-\frac{\left|y-\left(G_{2} \ldots G_{K}\right)\left(h_{1} \ldots h_{K}\right) x\right|^{2}}{(1+\rho) \sigma^{2}}\right\} d x\right]^{1+\rho} d y d h_{1} \ldots d h_{K}\right\}
\end{aligned}
$$

$$
\begin{aligned}
f_{K}(n, \gamma)=\operatorname{Pr}\left\{\gamma_{1} \geq \gamma, \gamma_{2} \geq \gamma, \ldots, \gamma_{K} \geq \gamma, \sum_{k=1}^{K-1} \gamma_{k} \geq(n-1) \gamma\right\} & \\
& +\operatorname{Pr}\left\{\gamma_{1} \geq \gamma, \gamma_{2} \geq \gamma, \ldots, \gamma_{K} \geq n \gamma-\sum_{k=1}^{K-1} \gamma_{k}, \sum_{k=1}^{K-1} \gamma_{k} \leq(n-1) \gamma\right\}
\end{aligned}
$$




\section{Proof of Theorem 1}

From Proposition 1, and using the PDF of $\gamma_{b}$ given by Proposition 2, we have

$$
\begin{aligned}
\tilde{E}_{0}(\rho)=-\ln & \left\{\int_{0}^{\infty}\left(1+\frac{\gamma}{1+\rho}\right)^{-\rho} p_{\gamma_{\mathrm{b}}}(\gamma) d \gamma\right\} \\
=-\ln & \left\{\int _ { 0 } ^ { \infty } ( 1 + \frac { \gamma } { 1 + \rho } ) ^ { - \rho } \left(\sum_{k=0}^{K-2} \frac{\left(K^{2}-K\right)^{k} K}{k ! \bar{\gamma}^{k+1}} \gamma^{k}\right.\right. \\
+ & \left.\left.\frac{\left(K^{2}-K\right)^{K-1} K^{2}}{(K-1) ! \bar{\gamma}^{K}} \gamma^{K-1}\right) e^{\frac{-K^{2} \gamma}{\bar{\gamma}}} d \gamma\right\} .
\end{aligned}
$$

Clearly $\tilde{E}_{0}(\rho)=0$ for $\rho=0$. We next derive $\tilde{E}_{0}(\rho)$ for $0<\rho \leq 1$. Define

$$
\mathcal{I}_{n}(a, \rho, \mu) \triangleq \int_{0}^{\infty}(1+a x)^{-\rho} x^{n} e^{-\mu x} d x
$$

To evaluate the integral $\mathcal{I}_{n}(a, \rho, \mu)$, we first express $(1+a x)^{-\rho}$ in terms of Meijer's G-functions by using [22, eq. (8.4.2.5)] as follows

$$
(1+a x)^{-\rho}=\frac{1}{\Gamma(\rho)} G_{1,1}^{1,1}\left(\begin{array}{l|l}
a x & \begin{array}{l}
1-\rho \\
0
\end{array}
\end{array}\right) .
$$

Substituting (39) into (38), and evaluating the integral $\mathcal{I}_{n}(a, \rho, \mu)$ with the help of the identity [22, eq. (2.24.3.1)], we obtain

$$
\mathcal{I}_{n}(a, \rho, \mu)=\frac{1}{\Gamma(\rho) \mu^{n+1}} G_{2,1}^{1,2}\left(\begin{array}{l|l}
\frac{a}{\mu} & -n, 1-\rho \\
0
\end{array}\right) .
$$

From (37), (38), and (40), we derive (24) and complete the proof.

\section{Proof of Corollary 1}

From (16), the ergodic capacity can be derived from the RCEE as follows

$$
\langle C\rangle=\left.\frac{1}{K}\left[\frac{\partial \tilde{E}_{0}(\rho)}{\partial \rho}\right]\right|_{\rho=0}=\frac{1}{K} \int_{0}^{\infty} \ln (1+\gamma) p_{\gamma_{\mathrm{b}}}(\gamma) d \gamma .
$$

Similarly to the derivation of $\tilde{E}_{0}(\rho)$, we first evaluate

$$
\mathcal{J}_{n}(\mu) \triangleq \int_{0}^{\infty} \ln (1+x) x^{n} e^{-\mu x} d x
$$

by expressing $\ln (1+x)$ in terms of Meijer's G-function with the help of [22, eq. (8.4.6.5)] as

$$
\ln (1+x)=G_{2,2}^{1,2}\left(x \mid \begin{array}{l}
1,1 \\
1,0
\end{array}\right)
$$

and again using the identity [22, eq. (2.24.3.1)]. Then substituting $\mathcal{J}_{n}(\mu)$ into (41), we get (25).

\section{REFERENCES}

[1] M. O. Hasna and M.-S. Alouini, "Outage probability of multihop transmission over Nakagami fading channels," IEEE Commun. Lett., vol. 7, no. 5, pp. 216-218, May 2003.

[2] G. K. Karagiannidis, T. A. Tsiftsis, and R. K. Mallik, "Bounds for multihop relayed communications in Nakagami- $m$ fading," IEEE Trans. Commun., vol. 54, no. 1, pp. 18-22, Jan. 2006.

[3] G. K. Karagiannidis, "Performance bounds of multihop wireless communications with blind relays over generalized fading channels," IEEE Trans. Wireless Commun., vol. 5, no. 3, pp. 498-503, Mar. 2006.

[4] R. G. Gallager, Information Theory and Reliable Communication. New York: Wiley, 1968.

[5] T. Ericson, "A Gaussian channel with slow fading," IEEE Trans. Inf. Theory, vol. 16, no. 3, pp. 353-355, May 1970.

[6] W. K. M. Ahmed and P. J. McLane, "Random coding error exponents for two-dimensional flat fading channels with complete channel state information," IEEE Trans. Inf. Theory, vol. 45, no. 4, pp. 1338-1346, May 1999.

[7] H. Shin and M. Z. Win, "Gallager's exponent for MIMO channels: A reliability-rate tradeoff," IEEE Trans. Commun., vol. 57, no. 4, pp. 972 985, Apr. 2009.

[8] H. Q. Ngo, T. Q. S. Quek, and H. Shin, "Random coding error exponent for dual-hop Nakagami- $m$ fading channels with amplify-and-forward relaying," IEEE Commun. Lett., vol. 13, no. 11, pp. 823-825, Feb. 2009.

[9] _ - "Amplify-and-forward two-way relay networks: Error exponents and resource allocation," IEEE Trans. Commun., vol. 58, no. 9, pp. 2653-2666, Sep. 2010.

[10] S. V. Maiya and T. E. Fuja, "One-hop vs. two-hop routing in simple networks with fading: An outage probability analysis addressing spectral efficiency," in Proc. IEEE Wireless Commun. and Networking Conf. (WCNC'08), Las Vegas, USA, 2008, pp. 494-499.

[11] M. Sikora, J. N. Laneman, M. Haenggi, J. Daniel J. Costello, and T. Fuja, "On the optimum number of hops in linear wireless networks," in Proc. IEEE Information Theory Workshop (ITW'04), San Antonio, TX, Oct. 2004, pp. 165-169.

[12] A. Florea and H. Yanikomeroglu, "On the optimal number of hops in infrastructure-based fixed relay networks," in Proc. IEEE Global Communications Conf. (GLOBECOM'05), MO, USA, Nov. 2005, pp. 3242-3247.

[13] Ö. Oyman, J. N. Laneman, and S. Sandhu, "Multihop relaying for broadband wireless mesh networks: From theory to practice," IEEE Commun. Mag., vol. 45, no. 11, pp. 116-122, Nov. 2007.

[14] Ö. Oyman, and S. Sandhu, "A Shannon-theoretic perspective on fading multihop networks," in Proc. IEEE Information Sciences and Systems (CISS'06), Princeton, NJ, Mar. 2006, pp. 525-530.

[15] J. Wu, "Connectivity of mobile linear networks with dynamic node population and delay constraint," IEEE J. Sel. Areas Commun., vol. 27, no. 7, pp. 1218-1225, Sep. 2009.

[16] S. Panichpapiboon and W. Pattara-atikom, "Connectivity requirements for self-organizing traffic information systems," IEEE Trans. Veh. Technol., vol. 57, no. 6, pp. 3333-3340, Nov. 2008.

[17] M. Sikora, J. N. Laneman, M. Haenggi, D. J. Costello, and T. E. Fuja, "Bandwidth-and power-efficient routing in linear wireless networks," IEEE Trans. Inf. Theory, vol. 52, no. 6, pp. 2624-2632, Jun. 2006.

[18] M. O. Hasna and M.-S. Alouini, "A performance study of dual-hop transmissions with fixed gain relays," IEEE Trans. Wireless Commun., vol. 3, no. 6, pp. 1963-1968, Nov. 2004.

[19] _ _ "End-to-end performance of transmission systems with relays over Rayleigh-fading channels," IEEE Trans. Wireless Commun., vol. 2, no. 6 , pp. 1126-1131, Nov. 2003.

[20] 1 , "Harmonic mean and end-to-end performance of transmission systems with relays," IEEE Trans. Commun., vol. 52, no. 1, pp. 130-135, Jan. 2004.

[21] P. A. Anghel and M. Kaveh, "Exact symbol error probability of a cooperative network in a Rayleigh-fading environment," IEEE Trans. Wireless Commun., vol. 3, no. 5, pp. 1416-1421, Sep. 2004.

[22] A. P. Prudnikov, Y. A. Brychkov, and O. I. Marichev, Integrals and Series. New York: Gordon and Breach Science, 1990, vol. 3.

[23] W. K. M. Ahmed, "Information theoretic reliability function for flat fading channels," Ph.D. dissertation, Queen's University, Kingston, ON, Canada, Sept. 1997. 


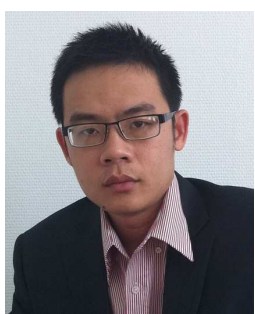

Hien Quoc Ngo received the B.S. degree in Electrical Engineering, major Telecommunications from Ho Chi Minh City University of Technology, Vietnam, in 2007, and the M.S. degree in Electronics and Radio Engineering from Kyung Hee University, Korea, in 2010. From 2008 to 2010, he was with the Communication and Coding Theory Laboratory, Kyung Hee University, where he did research on wireless communication and information theories, in particular are cooperative communications, game theory and network connectivity. Since April 2010, he is a Ph.D. student of the Division for Communication Systems in the Department of Electrical Engineering (ISY) at Linköping University (LiU) in Linköping, Sweden. His current research interests include MIMO systems with very large antenna arrays, cooperative communications, and interference networks.

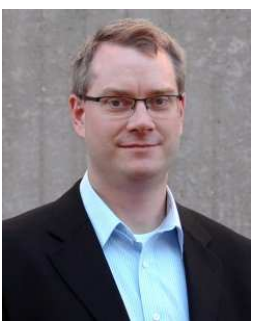

Erik G. Larsson received his Ph.D. degree from Uppsala University, Sweden, in 2002. Since 2007, he is Professor and Head of the Division for Communication Systems in the Department of Electrical Engineering (ISY) at Linköping University (LiU) in Linköping, Sweden. He has previously been Associate Professor (Docent) at the Royal Institute of Technology (KTH) in Stockholm, Sweden, and Assistant Professor at the University of Florida and the George Washington University, USA.

His main professional interests are within the areas of wireless communications and signal processing. He has published some 70 journal papers on these topics, he is co-author of the textbook SpaceTime Block Coding for Wireless Communications (Cambridge Univ. Press, 2003) and he holds 10 patents on wireless technology.

$\mathrm{He}$ is Associate Editor for the IEEE Transactions on Communications and he has previously been Associate Editor for several other IEEE journals. $\mathrm{He}$ is a member of the IEEE Signal Processing Society SAM and SPCOM technical committees. He is active in conference organization, most recently as the Technical Chair of the Asilomar Conference on Signals, Systems and Computers 2012 and Technical Program co-chair of the International Symposium on Turbo Codes and Iterative Information Processing 2012. 